\title{
Effects of dexrazoxane on doxorubicin- related cardiotoxicity and second malignant neoplasms in children with osteosarcoma: a report from the Children's Oncology Group
}

Lisa M. Kopp ${ }^{1,2^{*}}$ D, Richard B. Womer ${ }^{3}$, Cindy L. Schwartz ${ }^{4}$, David H. Ebb ${ }^{5}$, Vivian I. Franco ${ }^{6}$, David Hall', Donald A. Barkauskas ${ }^{7,8}$, Mark D. Krailo ${ }^{7,8}$, Holcombe E. Grier ${ }^{9}$, Paul A. Meyers ${ }^{10}$, Leonard H. Wexler ${ }^{10}$, Neyssa M. Marina ${ }^{11}$, Katherine A. Janeway ${ }^{9}$, Richard Gorlick ${ }^{12}$, Mark L. Bernstein ${ }^{13}$, Steven E. Lipshultz ${ }^{6}$ and for the Children's Oncology Group

\begin{abstract}
Background: Dexrazoxane protects from lower-cumulative-dose doxorubicin cardiotoxicity, but the effect of dexrazoxane in children with sarcoma treated with higher-cumulative-dose doxorubicin is unknown.

Methods: We evaluated children with osteosarcoma (OS) on two Children's Oncology Group trials with higher dose doxorubicin $\left(375-600 \mathrm{mg} / \mathrm{m}^{2}\right)$ preceded by dexrazoxane (10:1 dexrazoxane:doxorubicin dosing). They were evaluated after the minimum expected treatment time (METT), defined as 28 weeks. Cardiotoxicity was identified by echocardiography and serum N-terminal pro-brain natriuretic peptide (NT-proBNP). Second malignant neoplasm (SMN) data was collected.

Results: All children had normal left ventricular (LV) systolic function as measured by LV fractional shortening and no heart failure. The end-diastolic septal thickness $Z$-scores $(P<0.01)$ and LV mass $Z$-scores $(P<0.01)$ were significantly smaller than normal for body-surface area in both sexes. The average LV mass $Z$-scores were significantly smaller for girls $(P<0.01)$ and marginally smaller for boys $(P=0.06)$. Girls had significantly smaller LV end-diastolic dimension Z-scores normalized to BSA $(P<0.01)$ compared to healthy controls and had significant increases in NT-proBNP. Four children developed SMNs as first events, a rate similar to historical controls.

\footnotetext{
* Correspondence: Ikopp@email.arizona.edu

Preliminary data from this study was presented as abstracts at the June 1-5, 2012 (Abstract \#9503) and at the June 2-6, 2017 (Abstract \#10519) ASCO Annual Meetings.

${ }^{1}$ Department of Epidemiology and Biostatistics, Mel and Enid Zuckerman College of Public Health, The University of Arizona, 1295 N Martin Ave. PO Box 245210, Tucson, AZ 85724, USA

${ }^{2}$ University of Arizona Cancer Center, University of Arizona, Tucson, AZ, USA

Full list of author information is available at the end of the article
}

(c) The Author(s). 2019 Open Access This article is distributed under the terms of the Creative Commons Attribution 4.0 International License (http://creativecommons.org/licenses/by/4.0/), which permits unrestricted use, distribution, and reproduction in any medium, provided you give appropriate credit to the original author(s) and the source, provide a link to the Creative Commons license, and indicate if changes were made. The Creative Commons Public Domain Dedication waiver (http://creativecommons.org/publicdomain/zero/1.0/) applies to the data made available in this article, unless otherwise stated. 
(Continued from previous page)

Conclusions: Dexrazoxane prevented LV dysfunction and heart failure in children with OS receiving higher dose doxorubicin. However, LV structural changes were not fully prevented, especially in girls. As a result, hearts become abnormally small for body size, resulting in higher LV stress. Dexrazoxane did not increase the risk of SMN. Dexrazoxane should be used in this population, particularly for girls, to mitigate anthracycline-induced cardiotoxicity.

Trial registrations: ClinicalTrials.gov: NCT00003937 (P9754) registered 1 Nov 1999, and NCT00023998 (AOST0121) registered 13 Sept 2001.

Keywords: Osteosarcoma, Pediatrics, Cardiotoxicity, Doxorubicin,

\section{Background}

Osteosarcoma (OS) is the most common malignant bone tumor in children and adolescents. Multi-agent chemotherapy and surgery have greatly improved survival for patients with localized disease [1,2]. Doxorubicin is perhaps the most important agent for the treatment of OS [3]. Most protocols use cumulative doses of 375$450 \mathrm{mg} / \mathrm{m}^{2}$, which can be associated with cardiotoxicity during treatment, as well as long-term cardiovascular morbidity and early mortality $[4,5]$.

The Childhood Cancer Survivor Study (CCSS) reported cardiac death as the second most common cause of late mortality in childhood cancer survivors, particularly in those receiving high doses of anthracyclines [6, 7]. The hazard ratio of adverse cardiac outcomes in survivors who received $\geq 250 \mathrm{mg} / \mathrm{m}^{2}$ of an anthracycline was up to fivefold higher than in those receiving less anthracycline [6, 7]. These data rely on self-reported events and may not reflect the full spectrum of cardiac injury. While dexrazoxane mitigates anthracyclines' cardiotoxic effects [8, 9], reports of an increased risk of second malignant neoplasm (SMN) have limited its use in pediatric oncology $[10,11]$.

Two Children's Oncology Group (COG) trials for OS provide a large cohort for assessing the impact of dexrazoxane in children and adolescents treated with higher cumulative doses of doxorubicin $[12,13]$. We have previously published data from these trials on left ventricular (LV) systolic function (fractional shortening) and LV stress (N-terminal pro-brain natriuretic peptide, NTproBNP) $[12,13]$. However, we have also noted that late anthracycline-associated cardiotoxicity in long-term survivors of childhood ALL and OS results from two distinct pathways [14-18].

The first pathway is a direct effect of doxorubicin. Doxorubicin promotes formation of free radicals that disrupt cardiomyocyte cellular constituents, particularly in the mitochondria. This impairs the intrinsic health of the remaining cardiomyocytes and depresses contractility, while also causing cell death $[8,19]$.

The second pathway is the development of a restrictive cardiomyopathy due to LV dimensions and wall thickness that are abnormally small for body-surface area, resulting in increased LV afterload (stress) that may ultimately reduce LV function and lead to heart failure.

The objectives of the current analysis were to determine: 1) the incidence of cardiotoxicity in children and adolescents with OS receiving high cumulative dose doxorubicin $\left(450-600 \mathrm{mg} / \mathrm{m}^{2}\right)$ or trastuzumab plus dexrazoxane in the COG OS clinical trials P9754 and AOST0121 [12, 13], 2) the cumulative incidence of SMN.

\section{Methods \\ Treatment protocols}

Children, adolescents, and young adults with measurable, newly diagnosed, high-grade OS were enrolled on one of two multi-institutional COG OS protocols: P9754 between 1999 and 2002 [12, 13], and on AOST0121 between 2001 and 2005. Written informed consent was obtained from children and/or their guardians according to local institutional review board guidelines before starting therapy.

P9754 [13] included three sequential phase II pilot studies with a complex design for localized OS. Treatment was based on MAP (methotrexate, doxorubicin, cisplatin), with or without added ifosfamide, ifosfamideetoposide (IE), or intensification of doxorubicin (cumulative dose $600 \mathrm{mg} / \mathrm{m}^{2}$ ) in patients whose tumor showed a poor histologic response. AOST0121 [12] was a phase II study for metastatic OS; patients whose tumors had overexpression of human epidermal growth factor receptor 2 (HER2) received MAPIE with trastuzumab, while the other patients received MAP.

Both studies required normal LV function (LV fractional shortening of $\geq 28 \%$ or LV ejection fraction $\geq 50 \%$ ) at enrollment. Patients with a history of pericarditis, myocarditis, and symptomatic dysrhythmias or conduction disturbances were excluded.

\section{Therapy}

All patients received MAP with dexrazoxane given in a 10:1 ratio before each dose of doxorubicin. Surgical 
Table $1 \mathrm{SMN}^{\mathrm{a}}$ Risk

\begin{tabular}{|c|c|c|c|c|}
\hline Treatment Arm & Dexrazoxane & Doxorubicin & Ifosfamide & Etoposide \\
\hline AOST0121 HER2+ & $3.75 \mathrm{~g} / \mathrm{m}^{2}$ & $375 \mathrm{mg} / \mathrm{m}^{2}$ & $51 \mathrm{~g} / \mathrm{m}^{2}$ & $1.5 \mathrm{~g} / \mathrm{m}^{2}$ \\
\hline AOST0121 HER2- & $3.75 \mathrm{~g} / \mathrm{m}^{2}$ & $375 \mathrm{mg} / \mathrm{m}^{2}$ & $51 \mathrm{~g} / \mathrm{m}^{2}$ & $1.5 \mathrm{~g} / \mathrm{m}^{2}$ \\
\hline P9754 Pilot 1 Good Response & $4.5 \mathrm{~g} / \mathrm{m}^{2}$ & $450 \mathrm{mg} / \mathrm{m}^{2}$ & & \\
\hline P9754 Pilot 1 Standard Response & $6 \mathrm{~g} / \mathrm{m}^{2}$ & $600 \mathrm{mg} / \mathrm{m}^{2}$ & & \\
\hline P9754 Pilot 2 Good Response & $4.5 \mathrm{~g} / \mathrm{m}^{2}$ & $450 \mathrm{mg} / \mathrm{m}^{2}$ & $27 \mathrm{~g} / \mathrm{m}^{2}$ & \\
\hline P9754 Pilot 2 Standard Response & $6 \mathrm{~g} / \mathrm{m}^{2}$ & $600 \mathrm{mg} / \mathrm{m}^{2}$ & $45 \mathrm{~g} / \mathrm{m}^{2}$ & \\
\hline P9754 Pilot 3 Good Response & $4.5 \mathrm{~g} / \mathrm{m}^{2}$ & $450 \mathrm{mg} / \mathrm{m}^{2}$ & $27 \mathrm{~g} / \mathrm{m}^{2}$ & \\
\hline P9754 Pilot 3 Standard Response & $4.5 \mathrm{~g} / \mathrm{m}^{2}$ & $450 \mathrm{mg} / \mathrm{m}^{2}$ & $60 \mathrm{~g} / \mathrm{m}^{2}$ & $1.5 \mathrm{~g} / \mathrm{m}^{2}$ \\
\hline
\end{tabular}

${ }^{a} S M N$ second malignant neoplasms

resection of primary and metastatic sites was planned after week 10 of chemotherapy in both studies. Echocardiograms were to be obtained at baseline, at specified intervals during therapy, and after completing treatment. Blood was to be collected at baseline, before and after doxorubicin administration (30 h after doses 3, 5, 6, 7, and 8 on P9754 and 24 to $72 \mathrm{~h}$ after doses 3 and 5 on AOST0121), and at the end of treatment.

\section{Echocardiographic and biomarker measurements}

Cardiotoxicity was defined by echocardiographic criteria and by serum concentrations of cardiac troponin- $\mathrm{T}$ (cTnT), which measures injury to cardiomyocytes, and of N-terminal pro-brain natriuretic peptide (NTproBNP), which is elevated in cardiomyopathy associated with increased myocardial stress from ventricular pressure or volume overload [20-23]. Central re-analyses of the original echocardiogram tracings from both studies were performed using computer digital analysis with methods used previously to establish normative data in children [24]. Echocardiograms were only included in this analysis if they were centrally reviewed and the patient had completed therapy.

Serum cTnT levels were determined at a central laboratory using the Elecsys Troponin-T STAT Immunoassay
(Roche Diagnostics Corporation; sensitivity, $0.01 \mathrm{ng} / \mathrm{mL}$ ); any detectable amount was considered abnormal. Levels of NT-proBNP were measured by using the Elecsys immunoanalyzer (Roche Diagnostics; sensitivity, $5 \mathrm{pg} / \mathrm{mL}$ ); the cardiomyopathy risk threshold was defined as $\geq 100$ $\mathrm{pg} / \mathrm{mL}$, and the risk threshold for heart failure was defined as $\geq 400 \mathrm{pg} / \mathrm{mL}[25,26]$. Central investigators evaluating echocardiograms and cardiac biomarkers were blinded to the patients' clinical status and treatment assignments but were aware of the study objectives. Results were not reported to the care centers.

\section{Second malignant neoplasms}

SMN were identified through National Cancer Institutemandated adverse event reporting. The cumulative doses of drugs given that have been associated with increased SMN risk are shown in Table 1.

\section{Statistical methods}

The Proc Mixed Procedure in SAS 9.4 was used for all regression models with a compound symmetry correlation structure for repeated measures within patients. The NT-proBNP raw data was log-transformed for analysis.

Table 2 Patient Characteristics

\begin{tabular}{|c|c|c|c|c|c|c|c|c|c|}
\hline \multicolumn{2}{|c|}{ Study and Treatment Arm } & \multirow{2}{*}{$\begin{array}{l}\text { Mean Age } \\
\text { at } \\
\text { Enrollment }^{\mathrm{a}}\end{array}$} & \multicolumn{2}{|l|}{ Patient Sex ${ }^{\mathrm{b}}$} & \multirow[t]{2}{*}{ Total } & \multirow{2}{*}{$\begin{array}{l}\text { Expected } \\
\text { Weeks }\end{array}$} & \multirow[t]{2}{*}{ Dex $^{c}$} & \multirow[t]{2}{*}{ Doxorubicin } & \multirow[t]{2}{*}{ Trastuzumab } \\
\hline & & & Male & Female & & & & & \\
\hline \multirow[t]{2}{*}{ AOST0121 } & AOST0121 HER2 Negative & $15.1(3.6)$ & $33(60.0 \%)$ & $22(40.0 \%)$ & $55(100 \%)$ & 34 & $3.75 \mathrm{~g} / \mathrm{m}^{2}$ & $375 \mathrm{mg} / \mathrm{m}^{2}$ & $70 \mathrm{mg} / \mathrm{kg}$ \\
\hline & AOST0121 HER2 Positive & $13.7(2.7)$ & $19(47.5 \%)$ & $21(52.5 \%)$ & $40(100 \%)$ & 34 & $3.75 \mathrm{~g} / \mathrm{m}^{2}$ & $375 \mathrm{mg} / \mathrm{m}^{2}$ & \\
\hline \multirow[t]{6}{*}{ P9754 } & P9754 Pilot 1 Good Response & $13.5(5.0)$ & $16(47.1 \%)$ & $18(52.9 \%)$ & $34(100 \%)$ & 28 & $4.5 \mathrm{~g} / \mathrm{m}^{2}$ & $450 \mathrm{mg} / \mathrm{m}^{2}$ & \\
\hline & P9754 Pilot 1 Standard Response & $13.9(4.4)$ & $48(62.3 \%)$ & $29(37.7 \%)$ & $77(100 \%)$ & 29 & $6 \mathrm{~g} / \mathrm{m}^{2}$ & $600 \mathrm{mg} / \mathrm{m}^{2}$ & \\
\hline & P9754 Pilot 2 Good Response & $13.9(3.9)$ & $7(33.3 \%)$ & $14(66.7 \%)$ & $21(100 \%)$ & 28 & $4.5 \mathrm{~g} / \mathrm{m}^{2}$ & $450 \mathrm{mg} / \mathrm{m}^{2}$ & \\
\hline & P9754 Pilot 2 Standard Response & $13.8(4.5)$ & $20(60.6 \%)$ & $13(39.4 \%)$ & $33(100 \%)$ & 31 & $6 \mathrm{~g} / \mathrm{m}^{2}$ & $600 \mathrm{mg} / \mathrm{m}^{2}$ & \\
\hline & P9754 Pilot 3 Good Response & $14.3(4.0)$ & $12(63.2 \%)$ & $7(36.8 \%)$ & $19(100 \%)$ & 28 & $4.5 \mathrm{~g} / \mathrm{m}^{2}$ & $450 \mathrm{mg} / \mathrm{m}^{2}$ & \\
\hline & P9754 Pilot 3 Standard Response & $13.4(4.6)$ & $20(54.1 \%)$ & 17 (45.9\%) & $37(100 \%)$ & 36 & $4.5 \mathrm{~g} / \mathrm{m}^{2}$ & $450 \mathrm{mg} / \mathrm{m}^{2}$ & \\
\hline
\end{tabular}

\footnotetext{
${ }^{\mathrm{a}}$ Mean (SD), years
}

${ }^{\mathrm{b}}$ Number (\%)

'Dex, Dexrazoxane 
Table 3 Number of Patients with Data Available After METT ${ }^{\mathrm{a}}$ (28 weeks)

\begin{tabular}{|c|c|c|c|c|c|c|c|}
\hline Variable & $\begin{array}{l}\text { LV Fractional } \\
\text { Shortening Z- } \\
\text { scores }\end{array}$ & $\begin{array}{l}\text { LV End-Diastolic } \\
\text { Dimension Z-scores }\end{array}$ & $\begin{array}{l}\text { LV End-Diastolic Posterior } \\
\text { Wall Thickness Z-scores }\end{array}$ & $\begin{array}{l}\text { LV Thickness to } \\
\text { Dimension Ratio Z- } \\
\text { scores }\end{array}$ & $\begin{array}{l}\text { End-Diastolic Septal } \\
\text { Thickness Z-scores }\end{array}$ & $\begin{array}{l}\text { LV Mass } \\
\text { Z-scores }\end{array}$ & $\begin{array}{l}\text { NT- } \\
\text { proBNF }\end{array}$ \\
\hline Overall & $n=81$ & $n=78$ & $n=78$ & $n=82$ & $n=70$ & $n=74$ & $n=59$ \\
\hline AOST0121 & $n=8$ & $n=5$ & $n=5$ & $n=9$ & $n=5$ & $n=5$ & $n=15$ \\
\hline P9754 & $n=73$ & $n=73$ & $n=73$ & $n=73$ & $n=65$ & $n=69$ & $n=44$ \\
\hline
\end{tabular}

${ }^{\mathrm{a} M E T T}$ minimum expected treatment time

To adjust for growth-related changes, echocardiographic data were standardized by age (LV fractional shortening) or body-surface area (BSA; LV mass, LV dimension, septal thickness, and LV wall thickness). We calculated a $Z$-score of deviation from normal values based on data from 285 normal children and adolescents [25] in whom BSA ranged from 0.2 to $2.2 \mathrm{~m}^{2}$, who had normal height, weight, height-for-weight percentiles, and blood pressure; and had no evidence of cardiac or other disorders.

Since the times of echocardiographic and NT-proBNP measurements varied among protocols and patients, we defined a minimum expected treatment time (METT) of 28 weeks from enrollment. We then partitioned the follow-up assessment points equally according the interval from METT: 0-37 days, 38-81 days, and $>81$ days. No echocardiogram performed after disease progression or SMN was considered in the analysis.

Time to SMN was the number of days from enrollment to diagnosis of an SMN unless disease progression or death occurred first as a competing event. Patients not experiencing an event were considered censored at last contact. The cumulative incidence of SMNs was calculated using the methods of Gray [27]. The cumulative incidence of SMNs in the OS study, INT-0133 [1], which did not include dexrazoxane, was the basis of the historical comparison.

\section{Results}

\section{Patients}

A total of 316 patients from P9754 and AOST0121 were available for evaluation, with a male:female ratio of 1.2:1 and a mean age at enrollment of 13.7 years as seen in Table 2. The mean and median follow-up time was 73 months (77 months for P9754 and 53 months for AOST0121) and 80 months (82 months for P9754 and 55 months for AOST1521) respectively. Echocardiograms were available for central review from 81 patients; 68 had cTnT data available and 59 had NT-proBNP data available as shown in Table 3. There was a substantial amount of data missing from patients enrolled on both studies as shown in Table 4.

\section{Echocardiographic measurements}

We found no predictors of LV fractional shortening, LV end-diastolic posterior wall thickness, or LV thickness-todimension ratio $Z$-scores (a marker of pathologic adverse LV remodeling) for patients evaluated after the METT. However, the LV end-diastolic septal thickness $Z$-scores was significantly smaller than normal for BSA in both sexes $(P<0.01$; Table 5$)$ while the LV wall thickness and LV mass $Z$-scores were significantly smaller than normal for girls $(P<0.01$; Table 5$)$ and marginally smaller for boys but not statistically significant $(P=0.06$; Table 5$)$. A significant association between the assessment point (the follow-up points divided equally according to the interval from METT: $0-37$ days, $38-81$ days, and $>81$ days) and the patient's sex was found $(P<0.01$; Table 6$)$, with $\mathrm{LV}$ end-diastolic dimension $Z$-scores decreasing over time in girls and increasing over time in boys (Fig. 1).

\section{Cardiac biomarker assays}

None of the 68 children with biomarker data in either trial had detectable cTnT concentrations at any time.

Table 4 Number of Patients with Data Available and Number Excluded due to Missing Data ${ }^{a}$ After METT $^{\mathrm{b}}$

\begin{tabular}{|c|c|c|c|c|c|c|c|}
\hline Variable & $\begin{array}{l}\text { LV Fractional } \\
\text { Shortening Z-scores }\end{array}$ & $\begin{array}{l}\text { LV End-Diastolic } \\
\text { Dimension Z-scores }\end{array}$ & $\begin{array}{l}\text { LV End-Diastolic } \\
\text { Posterior Wall } \\
\text { Thickness Z-scores }\end{array}$ & $\begin{array}{l}\text { LV Thickness to } \\
\text { Dimension } \\
\text { Ratio Z-scores }\end{array}$ & $\begin{array}{l}\text { End-Diastolic Septal } \\
\text { Thickness Z-scores }\end{array}$ & LV Mass Z-scores & NT-proBNP \\
\hline \multirow[t]{2}{*}{ Overall } & $n=229$ & $n=224$ & $n=221$ & $n=228$ & $n=212$ & $n=214$ & $n=156$ \\
\hline & $(n=148)$ & $(n=146)$ & $(n=143)$ & $(n=146)$ & $(n=142)$ & $(n=140)$ & $(n=97)$ \\
\hline \multirow[t]{2}{*}{ AOST0121 } & $n=90$ & $n=85$ & $n=83$ & $n=89$ & $n=83$ & $n=82$ & $n=42$ \\
\hline & $(n=82)$ & $(n=80)$ & $(n=78)$ & $(n=80)$ & $(n=78)$ & $(n=77)$ & $(n=27)$ \\
\hline \multirow[t]{2}{*}{ P9754 } & $n=139$ & $n=139$ & $n=138$ & $n=139$ & $n=129$ & $n=132$ & $n=114$ \\
\hline & $(n=66)$ & $(n=66)$ & $(n=65)$ & $(n=66)$ & $(n=64)$ & $(n=63)$ & $(n=70)$ \\
\hline
\end{tabular}

${ }^{a}$ Number of patients with missing data in parenthesis for each variable

${ }^{\mathrm{b}}$ METT minimum expected treatment time 
Table 5 Echocardiographic and NT-proBNP measurements of Patients Evaluated After METT' ${ }^{\mathrm{a}}(28$ weeks)

\begin{tabular}{|c|c|c|c|c|c|}
\hline Measurement & Group & $\mathrm{N}$ & Mean & 95\% Confidence Interval for Mean & $p^{b}$ \\
\hline$\overline{N T-p r o B N P^{2}}$ & All Patients & 59 & $47.3^{c}$ & $(36.3,61.6)^{c}$ & $<0.01$ \\
\hline \multirow[t]{3}{*}{ LV End-Diastolic Posterior Wall Thickness Z-score } & All Patients & 78 & -0.29 & $(-0.60,0.02)$ & 0.06 \\
\hline & Female & 30 & -0.57 & $(-1.04,-0.09)$ & 0.02 \\
\hline & Male & 48 & -0.10 & $(-0.50,0.30)$ & 0.63 \\
\hline \multirow[t]{3}{*}{ End-Diastolic Septal Thickness Z-score } & All Patients & 70 & -0.84 & $(-1.2,-0.48)$ & $<0.01$ \\
\hline & Female & 27 & -1.20 & $(-1.76,-0.64)$ & $<0.01$ \\
\hline & Male & 43 & -0.60 & $(-1.06,-0.15)$ & 0.01 \\
\hline \multirow[t]{3}{*}{ LV Fractional Shortening Z-score } & All Patients & 81 & -0.19 & $(-0.54,0.17)$ & 0.30 \\
\hline & Female & 32 & -0.46 & $(-1.02,0.10)$ & 0.10 \\
\hline & Male & 49 & 0.00 & $(-0.46,0.47)$ & 0.99 \\
\hline \multirow[t]{3}{*}{ LV Mass Z-score } & All Patients & 74 & -0.74 & $(-1.06,-0.42)$ & $<0.01$ \\
\hline & Female & 29 & -1.23 & $(-1.71,-0.75)$ & $<0.01$ \\
\hline & Male & 45 & -0.39 & $(-0.80,0.01)$ & 0.06 \\
\hline
\end{tabular}

${ }^{\mathrm{a}} \mathrm{METT}$ minimal expected treatment time

${ }^{b}$ In the Z-score columns, a Z-score of 0 represents the average value for a healthy child of the same age. Each $P$-value for the Z-score measurements is from the two-sided hypothesis test with the null hypothesis $\mathrm{H}_{0}$ : the population mean for the Z-scores is 0 . (A Z-score of 0 represents the average value for a healthy child of the same age.) The $P$-value for NT-proBNP is from the two-sided hypothesis test with null hypothesis $\mathrm{H}_{0}$ : the population geometric mean is 100

${ }^{c}$ NT-proBNP is a raw score. Analysis for the NT-proBNP was done using a natural log transformation to stabilize the variance. The back-transformed NT-proBNP numbers are presented here for ease of interpretability. Due to the back transformations, these represent geometric means

Overall the values for NT-proBNP were below the level of concern for heart failure (less than $400 \mathrm{pg} / \mathrm{mL}$ ) for the 59 children and adolescents evaluated after the minimum expected treatment time $(P<0.01$; Table 5$)$. However, NT-proBNP concentrations were significantly associated with study (AOST0121 and P9754) $(P=0.02$; Table 6). Because of this association, further analyses were done separately for P9754 and AOST0121 (Table 6).

In P9754, the values of log [NT-proBNP] increased over time for both sexes $(P<0.01)$ and were significantly higher in girls than in boys $(P<0.01)$ (Table 6$)$. However, there was no significant interaction between assessment point and sex $(P=0.59$; Table 6), represented by the common slope in Fig. 2. No P9754 patients had log [NT-proBNP] measurements which were in the range of heart failure risk (Fig. 2).

For AOST0121, patient sex $(P=0.03)$, assessment point $(P=0.05)$, and the interaction term between sex and assessment point $(P=0.03)$ were all found to be significant in the final model for log [NT-proBNP] (Table 6). The final model showed boys' log [NT-proBNP] decreasing over time $(P=0.03)$ and girls' log [NTproBNP] increasing over time $(P=0.08)$ (Table 6). One girl on AOST0121 had a log [NT-proBNP] value in the range of heart failure risk (Figure 3).

For a complete view of additional cardiac data, Table 7 reports all collected echocardiographic $Z$-scores and NT-proBNP measurements after METT.

\section{Second malignant neoplasms}

In AOST0121, 95 children and adolescents were included in the analysis and 6 patients were excluded: 1 was misdiagnosed for HER2 status and assigned the wrong treatment, 4 were ineligible for AOST0121, and 1 was excluded for both reasons. For P9754, 221 children and adolescents were included in the analysis, and 32 were excluded because they were declared ineligible during the study.

There were five patients with SMNs in P9754 and AOST0121 combined: 3 patients with acute myelogenous leukemia, 1 patient with myelodysplastic syndrome, and 1 patient with juvenile myelomonocytic leukemia. Four were first events and one occurred after relapse of osteosarcoma. Three of the patients were enrolled on AOST0121 and as part of the protocol were scheduled to receive etoposide with ifosfamide. The other two patients were enrolled on P9754, Pilot 2, and were not scheduled to receive etoposide and ifosfamide. The fiveyear cumulative incidence of SMNs was 1.3\% (95\% CI, 0.44 to $3.2 \%$ ). This was similar to the $1.7 \%$ five-year SMNs cumulative incidence in INT-0133 (95\% CI, 0.93 to $2.9 \%)(P=0.65)$, which did not use dexrazoxane (Figure 4).

\section{Discussion}

We reviewed data on 315 patients enrolled on two COG OS trials (P9754 and AOST0121), all of whom received higher cumulative dose doxorubicin with dexrazoxane cardioprotection. None of the 315 patients enrolled on either study had reported clinical cardiac toxicity after treatment with $450-600 \mathrm{mg} / \mathrm{m}^{2}$ of doxorubicin as per NCI-CTCAE (Common Terminology Criteria for Adverse Events) version 2, with or without trastuzumab 
Table 6 Cardiac and NT-proBNP Measurements

\begin{tabular}{|c|c|}
\hline Prediction Factor, Model & $p^{a}$ \\
\hline \multicolumn{2}{|l|}{ Left Ventricular End-Diastolic Dimension Z-Scores } \\
\hline \multicolumn{2}{|l|}{ Univariate Model } \\
\hline Sex & 0.04 \\
\hline \multicolumn{2}{|l|}{ Final Model } \\
\hline Assessment Point ${ }^{\mathrm{b}}$ & 0.77 \\
\hline $\operatorname{Sex}^{2}$ & 0.74 \\
\hline Assessment Point* Sex Interaction Term & $<0.01$ \\
\hline Male Slope ${ }^{c}$ & 0.02 \\
\hline Female Slope ${ }^{c}$ & 0.02 \\
\hline \multicolumn{2}{|l|}{ NT pro-BNP, Both Studies Combined } \\
\hline Sex & 0.02 \\
\hline \multicolumn{2}{|l|}{ NT pro-BNP, P9754 } \\
\hline \multicolumn{2}{|l|}{ Univariate Models } \\
\hline Assessment Point & 0.02 \\
\hline Sex & 0.02 \\
\hline \multicolumn{2}{|l|}{ Final Model } \\
\hline Assessment Point & $<0.01$ \\
\hline Sex & $<0.01$ \\
\hline Assessment Point $*$ Sex Interaction Term ${ }^{d}$ & 0.59 \\
\hline \multicolumn{2}{|l|}{ NT pro-BNP, AOST0121 ${ }^{\mathrm{e}}$} \\
\hline \multicolumn{2}{|l|}{ Final Model } \\
\hline Assessment Point ${ }^{f}$ & 0.05 \\
\hline Sex & 0.03 \\
\hline Assessment Point * Sex Interaction Term & 0.03 \\
\hline Male Slope ${ }^{c}$ & 0.03 \\
\hline Female Slope $e^{c, f}$ & 0.08 \\
\hline
\end{tabular}

${ }^{a}$ All $P$-value are Type $3 p$-values from models produced by Proc Mixed in SAS 9.4, unless otherwise specified

${ }^{\mathrm{b}}$ The main effects in the final model were not significant, but were kept because their interaction term was significant

${ }^{C} P$-value obtained by a linear hypothesis $\mathrm{H} 0$ : Slope $=0$ using Proc Mixed in SAS 9.4

${ }^{\mathrm{d}}$ The interaction term for assessment point and sex was not found to be significant and was dropped from the final model

${ }^{\mathrm{e}}$ Neither assessment point nor sex were significant in their respective univariate models

${ }^{\mathrm{f}}$ Marginally significant

and concomitant dexrazoxane $[12,13]$. We also reviewed detailed cardiac function data of in 81 patients enrolled on the two trials who had such data available. Many patients, especially girls, had echocardiographic abnormalities or elevated concentrations of NT-proBNP after treatment. Left ventricular end-diastolic septal thickness and LV mass were significantly smaller for BSA than normal for both sexes. These echocardiographic changes persisted: one-third of children evaluated $>81$ days after the METT had significantly smaller LV posterior wall thicknesses for BSA than normal controls. Girls also had a significantly smaller LV dimension $Z$-score which indicates greater susceptibility to doxorubicin cardiotoxicity $[7,15,28,29]$.

Anthracyclines target topoisomerase II $\beta$ to cause DNA double-strand breaks, impairing transcription and translation, and they also couple with iron to generate reactive oxygen species. These species, in turn, cause an irreversible cardiomyocytic mitochondriopathy $[8,9]$. Dexrazoxane chelates myocardial iron, preventing it from coupling with anthracyclines, thus preventing the mitochondriopathy [19].

Dexrazoxane is an effective cardioprotectant in children with acute lymphoblastic leukemia (ALL) [25, 3032]. In an early use of dexrazoxane in children with sarcomas, 38 children were randomly assigned to receive doxorubicin with or without dexrazoxane [33]. Those children receiving dexrazoxane were less likely to have subclinical cardiotoxicity and had smaller declines in LV ejection fraction [33]. In the current cohort of patients with OS, none experienced clinical heart failure [12, 13], and none had any marked changes in LV fractional shortening $Z$-scores (Table $5 P=0.30$ ), or in LV end diastolic septal thickness $Z$-scores (Table $5 P<0.01$ ) despite treatment with trastuzumab and/or with cumulative doxorubicin doses up to $600 \mathrm{mg} / \mathrm{m}^{2}$. Although our study was not randomized, we believe the minimal cardiac changes noted are likely attributable to the significant cardioprotective benefit of dexrazoxane.

In studies of women with breast cancer, trastuzumab plus doxorubicin had rates of clinical cardiotoxicity as high as $27 \%$ [34]. Trastuzumab alone had a relative risk of 5.11 for severe heart failure in a meta-analysis of women with breast cancer [35]. A retrospective study of women with HER2positive breast cancer who received doxorubicin and trastuzumab showed that the $25 \%$ who received dexrazoxane had significantly fewer cardiac events [36]. Our study supports this impression that dexrazoxane may mitigate the cardiotoxicity of doxorubicin given with trastuzumab.

In the $>200$ children with ALL randomly assigned to receive doxorubicin alone or with dexrazoxane, cTnT and NT-proBNP concentrations increased significantly in children who received doxorubicin alone [31]. These increases were related to abnormal echocardiographic findings 4 years later [25]. In the OS studies reported here, all patients received dexrazoxane, and none of the evaluable children and adolescents had elevated concentrations of cTnT. The NT-proBNP concentrations overall did not reach levels of concern for heart failure risk in either study, with only one girl having a value in the range of heart failure risk (Figures 2 and 3). In P9754, NT-proBNP concentrations were significantly higher in girls than in boys at the end of therapy and remained high but never indicated heart failure (Figure 2). In AOST0121 we had few NT-proBNP measurements available for analysis. At the end of therapy some 
Left Ventricular End-diastolic Dimension Z score v Assess ment Point by Sex

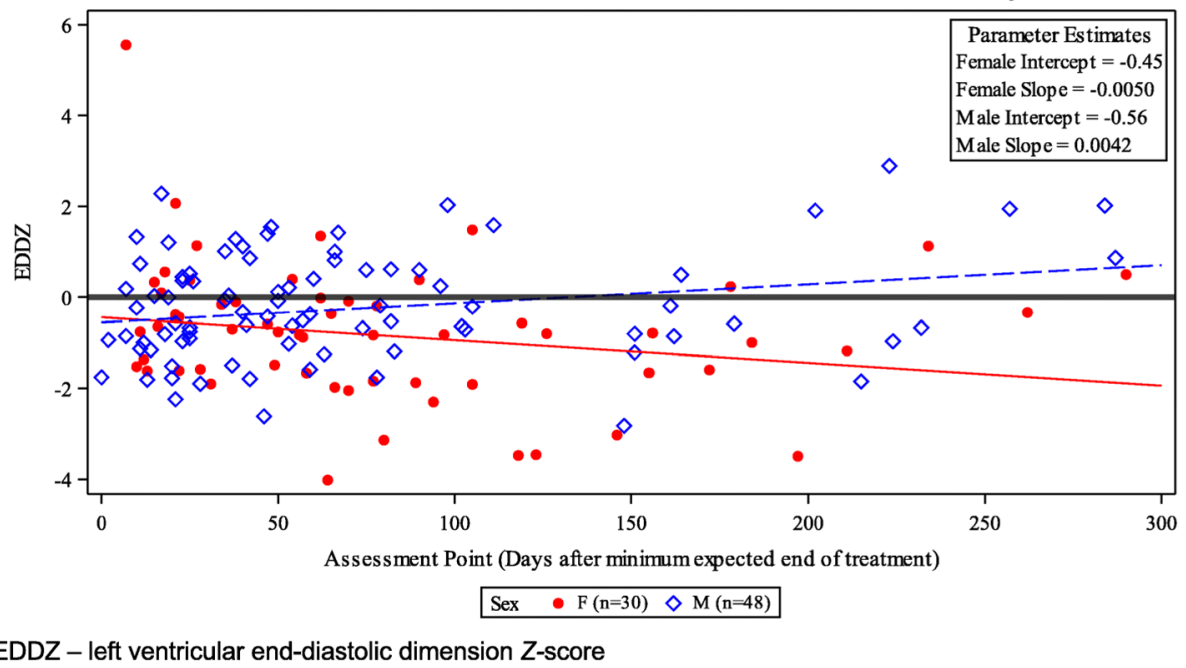

Fig. 1 Changes in Left Ventricular End-Diastolic Dimension Z-scores in Patients, by Sex

measurements were elevated for boys, which decreased with time. The findings in boys are consistent with the acute cardiotoxicity profile of trastuzumab, as the cardiotoxicity is often reversible once it is stopped [34, 35, 37, 38]. In the girls NT-proBNP slightly increased yet overall the concentration did not reach cardiomyopathy risk (Fig. 3).
Our finding that girls had more progressive abnormalities of LV structure (decreased LV wall thickness $Z$-scores, LV mass $Z$-scores and LV end-diastolic dimension $Z$-scores) than boys indicates that doxorubicintreated girls have hearts that are disproportionately small for body size, increasing their ventricular stress as indicated by a statistically significant increase in NT-

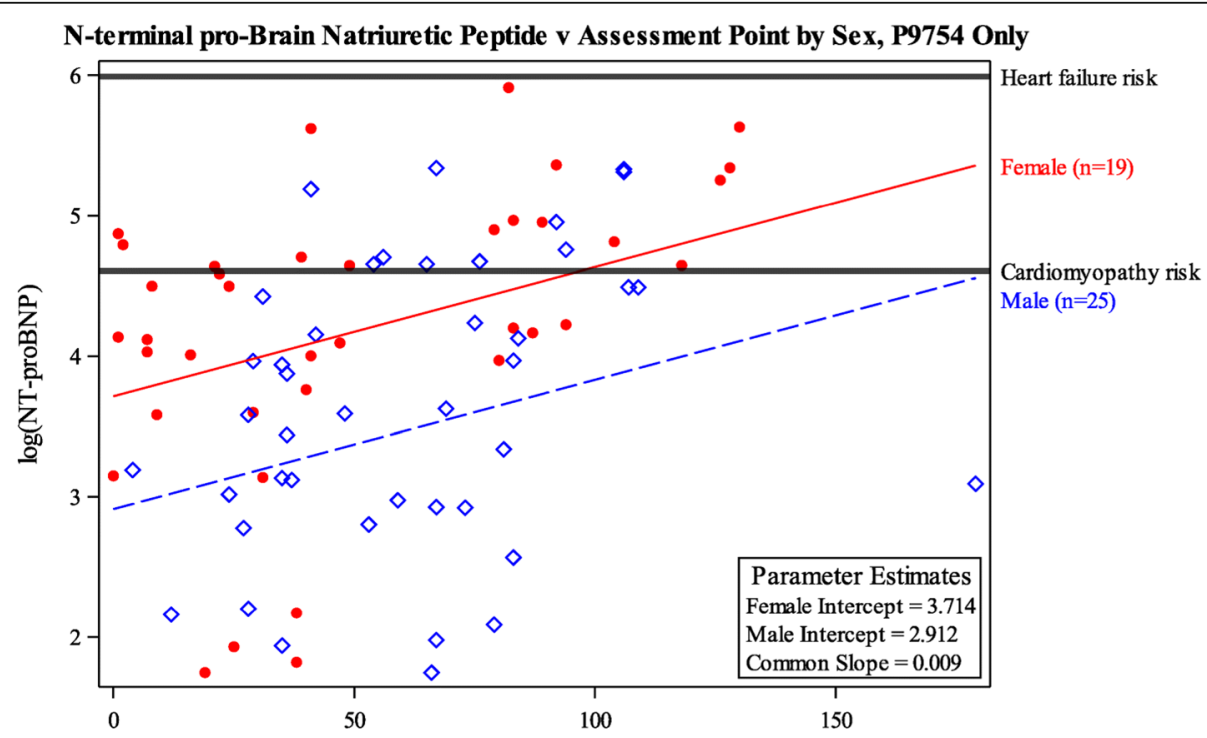

Assessment Point (Days after minimum expected end of treatment)

Sex Female $(\mathrm{n}=19) \diamond$ Male $(\mathrm{n}=25)$

Fig. 2 Risk of Cardiomyopathy and Heart Failure, by NT-proBNP, among Patients Enrolled on P9754, by Time Since Completing Treatment and Sex. Cardiomyopathy and heart failure risk thresholds for this NT-proBNP assay in children with cardiomyopathy have been determined to be $\geq 100 \mathrm{pg} / \mathrm{mL}$ and $\geq 400 \mathrm{pg} / \mathrm{mL}$, respectively $[25,26]$. The $y$-axis of this figure shows the log transformed values of NT-proBNP. The horizontal lines indicating cardiomyopathy and heart failure thresholds correspond to $100 \mathrm{pg} / \mathrm{mL}$ and $400 \mathrm{pg} / \mathrm{mL}$, respectively before they were log transformed 


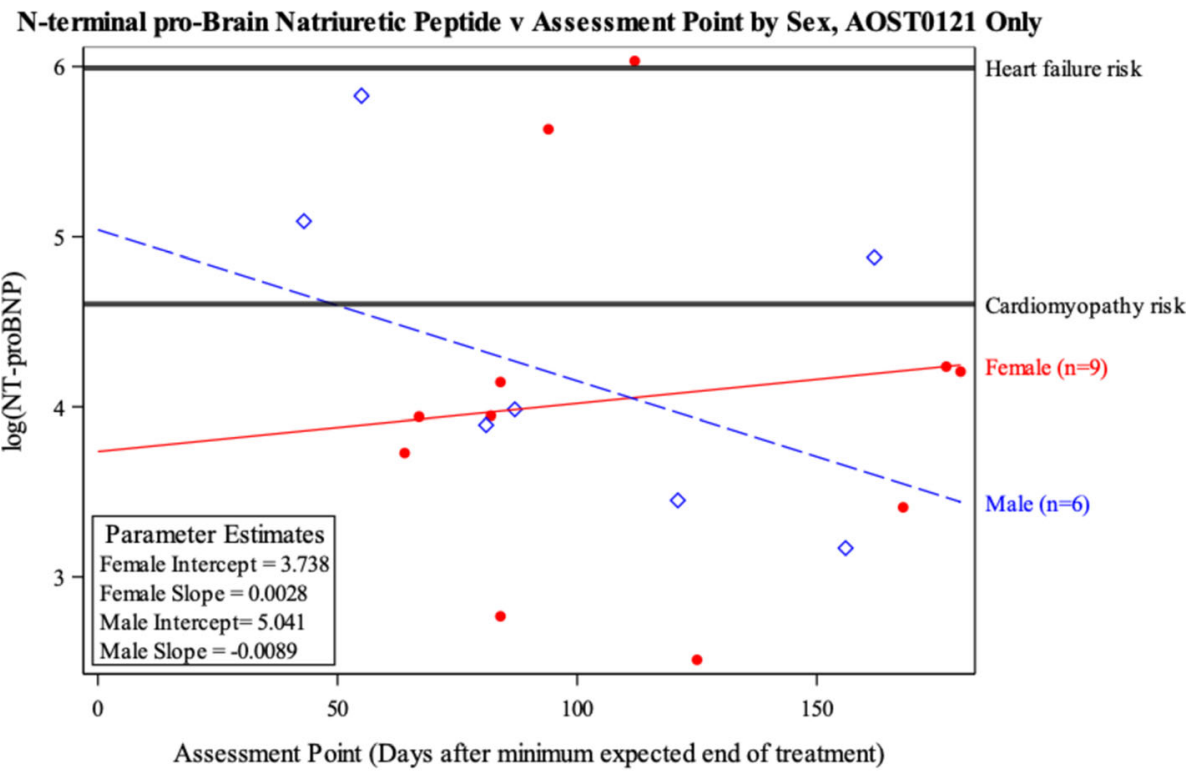

Sex $\bullet$ Female $(\mathrm{n}=9) \diamond$ Male $(\mathrm{n}=6)$

Fig. 3 Risk of Cardiomyopathy and Heart Failure, as Assessed by by NT-proBNP, among Patients enrolled on the COG AOST0121 Study, by Sex.. Cardiomyopathy was defined by having a NT-proBNP $\geq 100 \mathrm{pg} / \mathrm{mL}$ and risk of heart failure was defined as an NT-proBNP $\geq 400 \mathrm{pg} / \mathrm{mL}[25,26]$. The $y$-axis of this figure shows the log [NT-proBNP]. The horizontal lines indicating cardiomyopathy and heart failure thresholds correspond to $100 \mathrm{pg} / \mathrm{mL}$ and $400 \mathrm{pg} / \mathrm{mL}$, respectively before they were log transformed

proBNP concentration. These results indicate that the dexrazoxane's cardioprotection was incomplete for girls as reflected by the gender difference correlating with the smaller and more vulnerable hearts of girls. Other studies have also reported that female sex is an independent risk factor for late cardiac effects $[7,15,28]$.

The 5-year cumulative incidence of SMNs in P9754 and AOST0121 combined was similar to historical controls from the INT-0133 study (Fig. 4). Doxorubicin itself is shown to increase SMN risk, as highlighted in a review of $>6000$ childhood solid tumor survivors, which found a doxorubicin-dose-dependent increased SMN risk [39].

Some clinicians have hesitated to use dexrazoxane in children and adolescents with cancer because of a reported possible association between dexrazoxane and an increased risk of SMNs in patients with Hodgkin's lymphoma [10]. In those studies, uniquely, three topoisomerase inhibitors (etoposide, doxorubicin, and dexrazoxane) were used simultaneously. Chow et al. reviewed these same Hodgkin's lymphoma studies (POG 9425 and 9426), and the POG 9404 T-cell lymphoblastic lymphoma/leukemia study. With longer follow-up there was no increase in secondary AML/MDS attributable to dexrazoxane use [40]. In a multicenter study of 205 patients with high-risk ALL, half of whom received dexrazoxane with doxorubicin chemotherapy, dexrazoxane did not compromise the efficacy of doxorubicin [31, 32,
40], and there was no increase in the cumulative incidence of SMNs associated with dexrazoxane after a median follow-up of 6.2 years [41]. Similar findings were seen in other studies [30, 42], one of which examined 15,532 anthracycline-treated pediatric cancer patients, of whom 1406 received dexrazoxane, and found no increased risk of secondary AML [42]. In yet another set of sequential childhood protocols, among 553 highrisk ALL patients treated with dexrazoxane the only SMN was a single case of AML. The overall 5-year confidence interval of SMNs for patients was lower than the range in most historical studies, indicating SMNs were rare [43]. This updated SMN data led the European Medicines Agency (EMA) to withdraw its prohibition of dexrazoxane use in children aged 0-18 years. It now allows the use of dexrazoxane in Europe for children from the start of anthracycline chemotherapy if the planned cumulative dose is over $300 \mathrm{mg} / \mathrm{m}^{2}$. Their review also did not show evidence of dexrazoxane interference with chemotherapy, and led to removal of a safety warning for early death associated with dexrazoxane [44].

Our study was prospective, and all echocardiograms were centrally reviewed. However, the studies were not randomized so there were no comparison arms, and the numbers of children and adolescents with available data after the expected completion of treatment were small (echo data is missing in 74\%, troponin in $78 \%$ and BNP 
Table 7 Echocardiographic and NT-proBNP of Patients After METT ${ }^{\mathrm{a}}\left(28\right.$ weeks) ${ }^{\mathrm{b}}$

\begin{tabular}{|c|c|c|c|c|c|c|c|}
\hline Variable & $\begin{array}{l}\text { LV Fractional } \\
\text { Shortening Z-scores }\end{array}$ & $\begin{array}{l}\text { LV End-Diastolic } \\
\text { Dimension Z-scores }\end{array}$ & $\begin{array}{l}\text { LV End-Diastolic Posterior } \\
\text { Wall Thickness Z-scores }\end{array}$ & $\begin{array}{l}\text { LV Thickness to } \\
\text { Dimension Ratio } \\
\text { Z-scores }\end{array}$ & $\begin{array}{l}\text { End-Diastolic Septal } \\
\text { Thickness Z-scores }\end{array}$ & LV Mass Z-scores & NT-proBNP ${ }^{c}$ \\
\hline \multirow[t]{4}{*}{ Overall } & $\mathrm{n}=81$ & $n=78$ & $n=78$ & $n=82$ & $n=70$ & $n=74$ & $n=59$ \\
\hline & $\bar{x}=-0.19$ & $\bar{x}=-0.49$ & $\bar{x}=-0.29$ & $\bar{x}=-0.23$ & $\bar{x}=-0.84$ & $\bar{x}=-0.74$ & $\mathrm{GM}=47.3$ \\
\hline & $(-0.54,0.17)$ & $(-0.76,-0.21)$ & $(-0.60,0.02)$ & $(-0.53,0.07)$ & $(-1.2,-0.48)$ & $(-1.06,-0.42)$ & $(36.3,61.6)$ \\
\hline & $P=0.30$ & $P<0.01$ & $P=0.06$ & $P=0.13$ & $P<0.01$ & $P<0.01$ & $P<0.01$ \\
\hline \multicolumn{8}{|l|}{ Sex } \\
\hline \multirow[t]{4}{*}{ Female } & $n=32$ & $n=30$ & $n=30$ & $n=33$ & $n=27$ & $n=29$ & $n=28$ \\
\hline & $\bar{x}=-0.46$ & $\bar{x}=-0.82$ & $\bar{x}=-0.57$ & $\bar{x}=-0.29$ & $\bar{x}=-1.20$ & $\bar{x}=-1.23$ & $\mathrm{GM}=63.2$ \\
\hline & $(-1.02,0.10)$ & $(-1.24,-0.40)$ & $(-1.04,-0.09)$ & $(-0.75,0.16)$ & $(-1.76,-0.64)$ & $(-1.71,-0.75)$ & $(43.8,91.4)$ \\
\hline & $P=0.10$ & $P<0.01$ & $P=0.02$ & $P=0.20$ & $P<0.01$ & $P<0.01$ & $P=0.02$ \\
\hline \multirow[t]{4}{*}{ Male } & $n=49$ & $n=48$ & $\mathrm{n}=48$ & $\mathrm{n}=49$ & $n=43$ & $n=45$ & $n=31$ \\
\hline & $\bar{x}=0.00$ & $\bar{x}=-0.26$ & $\bar{x}=-0.10$ & $\bar{x}=-0.18$ & $\bar{x}=-0.60$ & $\bar{x}=-0.39$ & $\mathrm{GM}=36.1$ \\
\hline & $(-0.46,0.47)$ & $(-0.60,0.08)$ & $(-0.50,0.30)$ & $(-0.57,0.21)$ & $(-1.06,-0.15)$ & $(-0.80,0.01)$ & $(25.3,51.6)$ \\
\hline & $P=0.99$ & $P=0.14$ & $P=0.63$ & $P=0.36$ & $P=0.01$ & $P=0.06$ & $P<0.01$ \\
\hline \multicolumn{8}{|l|}{ Study } \\
\hline \multirow[t]{4}{*}{ AOST0121 } & $n=8$ & $n=5$ & $n=5$ & $n=9$ & $n=5$ & $n=5$ & $n=15$ \\
\hline & $\bar{x}=-0.70$ & $\bar{x}=-0.61$ & $\bar{x}=-0.64$ & $\bar{x}=0.10$ & $\bar{x}=-3.82$ & $\bar{x}=-0.86$ & $\mathrm{GM}=60.5$ \\
\hline & $(-2.06,0.67)$ & $(-1.82,0.59)$ & $(-2.06,0.78)$ & $(-0.97,1.17)$ & $(-5.05,-2.59)$ & $(-2.23,0.50)$ & $(35.0,104.6)$ \\
\hline & $P=0.31$ & $P=0.31$ & $P=0.37$ & $P=0.85$ & $P<0.01$ & $P=0.21$ & $P=0.07$ \\
\hline \multirow[t]{4}{*}{ P9754 } & $n=73$ & $n=73$ & $n=73$ & $n=73$ & $n=65$ & $n=69$ & $n=44$ \\
\hline & $\bar{x}=-0.15$ & $\bar{x}=-0.48$ & $\bar{x}=-0.27$ & $\bar{x}=-0.26$ & $\bar{x}=-0.63$ & $\bar{x}=-0.73$ & $\mathrm{GM}=43.9$ \\
\hline & $(-0.52,0.22)$ & $(-0.76,-0.20)$ & $(-0.59,0.04)$ & $(-0.57,0.05)$ & $(-0.96,-0.31)$ & $(-1.07,-0.40)$ & $(32.4,59.3)$ \\
\hline & $P=0.43$ & $P<0.01$ & $P=0.09$ & $P=0.10$ & $P<0.01$ & $P<0.01$ & $P<0.01$ \\
\hline \multicolumn{8}{|c|}{ Assessment Point ${ }^{d}$} \\
\hline \multirow[t]{4}{*}{0 to 37 days } & $n=48$ & $n=48$ & $n=45$ & $\mathrm{n}=45$ & $n=40$ & $n=38$ & $n=30$ \\
\hline & $\bar{x}=-0.18$ & $\bar{x}=-0.38$ & $\bar{x}=-0.40$ & $\bar{x}=-0.43$ & $\bar{x}=-0.39$ & $\bar{x}=-0.67$ & $\mathrm{GM}=32.6$ \\
\hline & $(-0.72,0.35)$ & $(-0.75,-0.01)$ & $(-0.85,0.05)$ & $(-0.90,0.03)$ & $(-0.80,0.03)$ & $(-1.13,-0.20)$ & $(18.4,57.7)$ \\
\hline & $P=0.49$ & $P=0.05$ & $P=0.08$ & $P=0.06$ & $P=0.07$ & $P<0.01$ & $P=0.01$ \\
\hline \multirow[t]{4}{*}{38 to 81 days } & $n=42$ & $n=40$ & $n=38$ & $n=41$ & $n=36$ & $n=32$ & $n=32$ \\
\hline & $\bar{x}=-0.13$ & $\bar{x}=-0.60$ & $\bar{x}=0.15$ & $\bar{x}=0.15$ & $\bar{x}=-0.82$ & $\bar{x}=-0.43$ & $\mathrm{GM}=45.2$ \\
\hline & $(-0.70,0.43)$ & $(-1.00,-0.21)$ & $(-0.33,0.64)$ & $(-0.33,0.63)$ & $(-1.24,-0.40)$ & $(-0.93,0.07)$ & $(26.4,77.3)$ \\
\hline & $P=0.64$ & $P<0.01$ & $P=0.53$ & $P=0.53$ & $P<0.01$ & $P=0.09$ & $P=0.01$ \\
\hline \multirow[t]{4}{*}{$>81$ days } & $n=39$ & $n=37$ & $n=37$ & $\mathrm{n}=40$ & $n=29$ & $n=36$ & $n=34$ \\
\hline & $\bar{x}=-0.24$ & $\bar{x}=-0.51$ & $\bar{x}=-0.57$ & $\bar{x}=-0.35$ & $\bar{x}=-1.46$ & $\bar{x}=-1.06$ & $\mathrm{GM}=67.6$ \\
\hline & $(-0.82,0.34)$ & $(-0.91,-0.10)$ & $(-1.05,-0.09)$ & $(-0.83,0.12)$ & $(-1.91,-1.02)$ & $(-1.53,-0.60)$ & $(39.9,114.5)$ \\
\hline & $P=0.41$ & $P=0.01$ & $P=0.02$ & $P=0.14$ & $P<0.01$ & $P<0.01$ & $P=0.11$ \\
\hline
\end{tabular}

${ }^{\mathrm{a}} M E T T$ minimal expected treatment time

${ }^{b}$ In the Z-score columns, a Z-score of 0 represents the average value for a healthy child of the same age

${ }^{c}$ NT-proBNP is a raw score. Analysis for the NT-proBNP was done using a natural log transformation to better adhere to the normality assumption for regression. The back-transformed NT-proBNP numbers are presented here for ease of interpretability. Due to the back transformations, these represent geometric means (GM). The $P$-value is from the two-sided hypothesis test with null hypothesis $\mathrm{H}_{0}$ : the population $\mathrm{GM}$ is 100

${ }^{\mathrm{d}}$ Assessment Point is the measure in days after the minimum expected treatment ( 28 weeks/196 days) and broken up into tertiles based on the 33.3 rd and 66.7th percentiles of the assessment points of all measurements. Due to multiple measurements per patient, it is possible for patients to be in multiple tertiles

in $81 \%$ of patients). Also, as the follow up period was short it is possible patients may have developed cardiotoxicity later in follow up. The substantial amount of missing data could have biased our results. Nonetheless, the absence of cardiotoxicity in children and adolescents receiving high-dose anthracycline therapy supports the conclusion that dexrazoxane is cardioprotective. There was no suggestion of an increased incidence in SMN. 


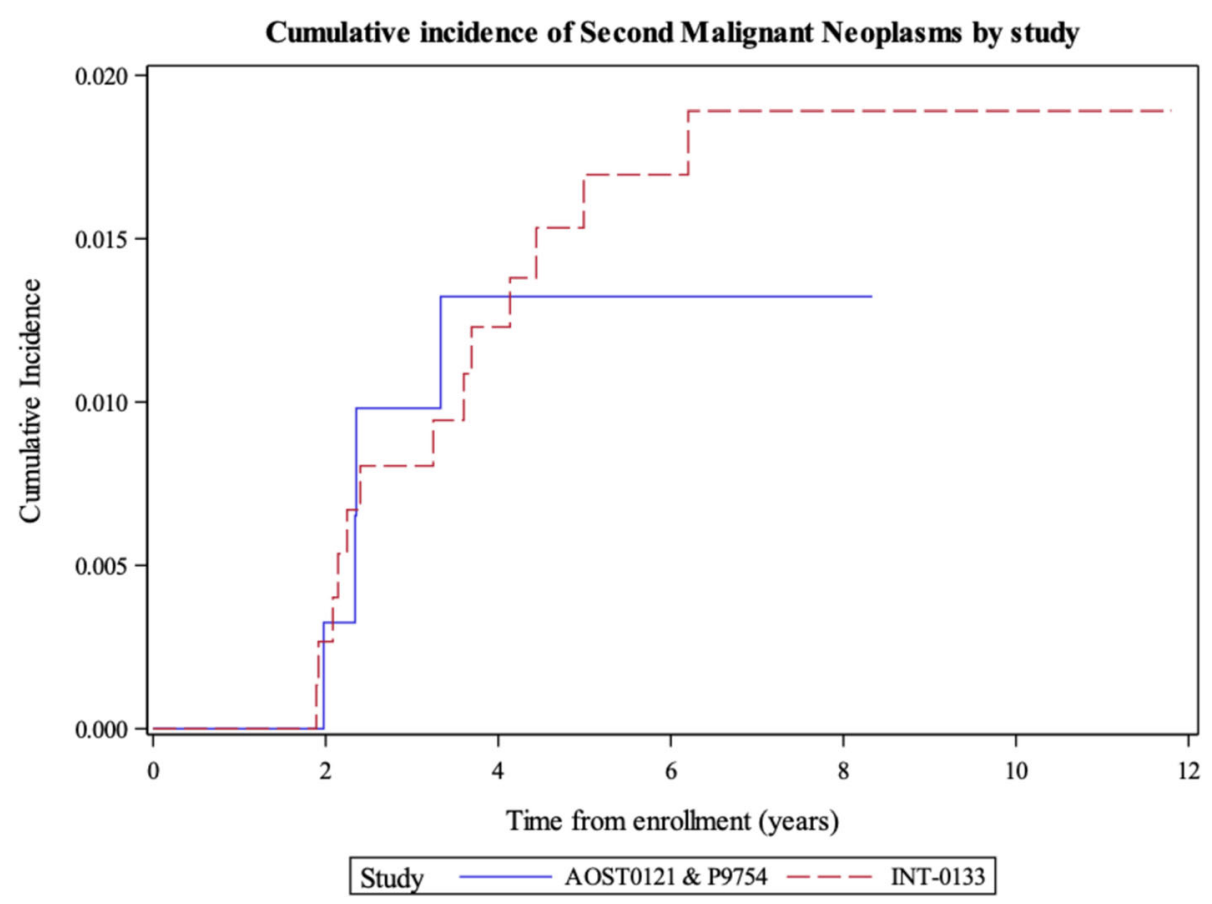

Fig. 4 5-year Cumulative Incidence of Second Malignant Neoplasms in The Clinical Trial INT-0133 versus COG P9754 and COG AOST0121

\section{Conclusions}

Our study adds to the literature derived from randomized trials that show evidence of dexrazoxane cardioprotection [30, 32, 33, 45]. As the number of childhood cancer survivors increases [46] the use of dexrazoxane may reduce the total cumulative burden for this vulnerable and medically complex population. Initial data from the ongoing COG ALTE11C2 protocol has demonstrated that with 16 years follow-up dexrazoxane is associated with statistically significant long-term cardioprotection, as evidenced by better LV function (fractional shortening) and lower levels of brain natriuretic peptides in multivariate analyses, when compared with anthracycline-treated patients who did not receive dexrazoxane [45]. Further, pharmacoeconomic analyses have indicated that the balance of additional costs driven by dexrazoxane compared with savings from a reduction in cardiology-associated costs and hospitalizations favored the use of dexrazoxane, a point that is important as we strive to reduce survivors' chronic health condition burden $[4,47]$.

Our data support the conclusion that dexrazoxane should be recommended for use in all children and adolescents with OS from the initiation of doxorubicin therapy, particularly in girls, who exhibit more cardiotoxicity than boys at equal cumulative doses. This is consistent with the new EMA indications and also takes into account that irreversible cardiomyocyte injury occurs from the first anthracycline dose, regeneration of cardiomyocytes is limited, drug therapy can only partially restore normal cardiac function, and the incidence of cardiac damage will increase with the duration of survival of pediatric cancer patients $[4,48]$.

\section{Abbreviations}

ALL: Acute lymphoblastic leukemia; AML: Acute myeloid leukemia; BSA: Body-surface area; COG: Children's Oncology Group; cTnT: Serum cardiac troponin T concentrations; EMA: European Medicines Agency; HER2: Human epidermal growth factor receptor 2; IE: Ifosfamide and etoposide; LV: Left ventricular; MAP: Methotrexate, doxorubicin, cisplatin; MDS: Myelodysplastic syndrome; METT: Minimum expected treatment time; NT-proBNP: Serum N-terminal pro-brain natriuretic peptide concentration; OS: Osteosarcoma; SMN: Second malignant neoplasm

\section{Acknowledgements}

We are grateful to all the children and their families that we have the privilege of caring for who participated in our studies. Also, we are very thankful to the study teams at the participating centers and St. Baldrick's Foundation. We are especially thankful to the QuadW Foundation for their continued support of osteosarcoma research.

\section{Authors' contributions}

LMK: study design, data collection, interpretation and manuscript preparation. RBM: study design, data collection, interpretation and manuscript preparation. CLS: study design, data collection, interpretation and manuscript preparation. DHE: study design, data collection, manuscript preparation. VIF: study design, data collection, manuscript preparation. DH: study design, data collection, interpretation and manuscript preparation. DAB: study design, data collection, interpretation and manuscript preparation. MDK: study design, data collection, interpretation and manuscript preparation. HEG: study design, data collection, manuscript preparation. PAM: study design, data collection, manuscript preparation. 
LHW: data collection, interpretation and manuscript preparation. NMM: study design, data collection, manuscript preparation. KAJ: study design, data collection, manuscript preparation. RG: study design, data collection, manuscript preparation. MLB: study design, data collection, interpretation and manuscript preparation. SEL: study design, data collection, interpretation and manuscript preparation. All authors read and approved the final version of the manuscript.

\section{Funding}

NCTN Operations Center Grant U10CA180886.

NCTN Statistics \& Data Center Grant U10CA180899.

St. Baldrick's Foundation supports the funding of Children's Oncology Group clinical trials. QuadW Foundation supported the funding of the statistical analysis for this study.

Funding for SL supported in part by grants from the National Institutes of Health (HL072705, HL078522, HL053392, CA127642, CA068484, HD052104, Al50274, HD052102, HL087708, HL079233, HL004537, HL087000, HL007188, HL094100, HL095127, and HD80002), Roche Diagnostics, Pfizer, the Children's Cardiomyopathy Foundation, Sofia's Hope, Inc., the Women's Cancer Association, the Lance Armstrong Foundation, the STOP Children's Cancer Foundation, the Scott Howard Fund, and the Michael Garil Fund.

\section{Availability of data and materials}

The data that support the findings of this study are available from Children's Oncology Group but are not publicly available. Data are however available from the authors upon reasonable request and with permission of the Children's Oncology Group.

\section{Ethics approval and consent to participate}

Ethical approval was obtained from each institution's institutional review board (IRB) prior to the study (AOST0121 or P9754) opening for accrual. Written informed consent was obtained from children, adolescents, adults and/or their guardians according to local IRB guidelines before starting therapy.

\section{Consent for publication}

Not applicable.

\section{Competing interests}

L.K. is an employee of the contract research organization Covance. M.K. is a consultant for Merck. K.J. has received travel reimbursement from Loxo oncology. R.G. has received laboratory research funding from Eisai. N.M. is an employee of Five Prime Therapeutics. P.M. has received travel reimbursement from Takeda and is a consultant for Eli Lilly and Astellas. S.L. is a consultant for Clinigen, a manufacturer of dexrazoxane, and Editor in Chief of Cardio-Oncology.

\section{Author details}

${ }^{1}$ Department of Epidemiology and Biostatistics, Mel and Enid Zuckerman College of Public Health, The University of Arizona, 1295 N Martin Ave. PO Box 245210, Tucson, AZ 85724, USA. University of Arizona Cancer Center, University of Arizona, Tucson, AZ, USA. ${ }^{3}$ Children's Hospital of Philadelphia, Philadelphia, PA, USA. ${ }^{4}$ Children's Hospital of Wisconsin, Medical College of Wisconsin, Milwaukee, WI, USA. ${ }^{5}$ Department of Pediatric Hematology-Oncology, Massachusetts General Hospital, Boston, MA, USA. ${ }^{6}$ Department of Pediatrics, University at Buffalo, Oishei Children's Hospital, Roswell Park Comprehensive Cancer Center, Buffalo, NY, USA. ${ }^{7}$ Children's Oncology Group, Monrovia, CA, USA. ${ }^{8}$ Department of Preventive Medicine, Keck School of Medicine, University of Southern California, Los Angeles, CA, USA. ${ }^{9}$ Dana-Farber Cancer Institute, Boston, MA, USA. ${ }^{10}$ Memorial Sloan Kettering Cancer Center, New York, NY, USA. ${ }^{11}$ Five Prime Therapeutics, Inc. South San Francisco, CA, USA. ${ }^{12}$ MD Anderson Cancer Center, Houston, TX, USA. ${ }^{13}$ IWK Health Centre, Halifax, Nova Scotia, Canada.

Received: 3 July 2019 Accepted: 10 September 2019

Published online: 28 October 2019

\section{References}

1. Meyers PA, et al. Osteosarcoma: a randomized, prospective trial of the addition of ifosfamide and/or muramyl tripeptide to cisplatin, doxorubicin, and high-dose methotrexate. J Clin Oncol. 2005;23(9):2004-11.
2. Bielack SS, et al. Prognostic factors in high-grade osteosarcoma of the extremities or trunk: an analysis of 1,702 patients treated on neoadjuvant cooperative osteosarcoma study group protocols. J Clin Oncol. 2002;20(3):776-90.

3. Smith MA, et al. Influence of doxorubicin dose intensity on response and outcome for patients with osteogenic sarcoma and Ewing's sarcoma. J Natl Cancer Inst. 1991;83(20):1460-70.

4. Bansal N, Amdani S, Lipshultz ER, Lipshultz SE. Chemotherapy-induced cardiotoxicity in children. Expert Opin Drug Metab Toxicol. 2017;13(8):81732. https://doi.org/10.1080/17425255.2017.1351547. Epub 2017 Jul 13. Review. PubMed PMID: 28679288

5. Getz KD, Sung L, Ky B, Gerbing RB, Leger KJ, Leahy AB, Sack L, Woods WG, Alonzo T, Gamis A, Aplenc R. Occurrence of Treatment-Related Cardiotoxicity and Its Impact on Outcomes Among Children Treated in the AAML0531 Clinical Trial: A Report From the Children's Oncology Group. J Clin Oncol. 2019; 37(1):12-21. https://doi.org/10.1200/JCO.18.00313. Epub 2018 Oct 31. PubMed PMID: 30379624; PubMed Central PMCID: PMC6354770.

6. Armstrong GT, et al. Late mortality among 5-year survivors of childhood cancer: a summary from the childhood Cancer survivor study. J Clin Oncol. 2009;27(14): 2328-38.

7. Mulrooney DA, et al. Cardiac outcomes in a cohort of adult survivors of childhood and adolescent cancer: retrospective analysis of the childhood Cancer survivor study cohort. BMJ. 2009;339:b4606.

8. Herman EH, et al. A review of the preclinical development of dexrazoxane. Prog Pediatr Cardiol. 2014;36(1):33-8.

9. Lipshultz SE, et al. Dexrazoxane for reducing anthracycline-related cardiotoxicity in children with cancer: an update of the evidence. Prog Pediatr Cardiol. 2014;36:39-40.

10. Tebbi CK, et al. Dexrazoxane-associated risk for acute myeloid leukemia/ myelodysplastic syndrome and other secondary malignancies in pediatric Hodgkin's disease. J Clin Oncol. 2007;25(5):493-500.

11. Walker DM, et al. Dexrazoxane use in pediatric patients with acute lymphoblastic or myeloid leukemia from 1999 and 2009: analysis of a national cohort of patients in the pediatric health information systems database. Pediatr Blood Cancer. 2013;60(4):616-20.

12. Ebb D, et al. Phase II trial of trastuzumab in combination with cytotoxic chemotherapy for treatment of metastatic osteosarcoma with human epidermal growth factor receptor 2 overexpression: a report from the children's oncology group. J Clin Oncol. 2012;30(20):2545-51.

13. Schwartz $\mathrm{CL}$, et al. Intensified chemotherapy with Dexrazoxane Cardioprotection in newly diagnosed nonmetastatic osteosarcoma: a report from the Children's oncology group. Pediatr Blood Cancer. 2016; 63(1):54-61.

14. Lipshultz SE, et al. Late cardiac effects of doxorubicin therapy for acute lymphoblastic leukemia in childhood. N Engl J Med. 1991;324(12):808-15.

15. Lipshultz SE, et al. Female sex and drug dose as risk factors for late cardiotoxic effects of doxorubicin therapy for childhood cancer. N Engl J Med. 1995; 332(26):1738-43.

16. Lipshultz SE, Colan SD. Cardiovascular trials in long-term survivors of childhood cancer. J Clin Oncol. 2004;22(5):769-73.

17. Lipshultz SE, et al. Chronic progressive cardiac dysfunction years after doxorubicin therapy for childhood acute lymphoblastic leukemia. J Clin Oncol. 2005;23(12):2629-36.

18. Lipshultz SE, Adams MJ. Cardiotoxicity after childhood cancer: beginning with the end in mind. J Clin Oncol. 2010;28(8):1276-81.

19. Lipshultz SE, et al. Impaired mitochondrial function is abrogated by dexrazoxane in doxorubicin-treated childhood acute lymphoblastic leukemia survivors. Cancer. 2016;122(6):946-53.

20. Cardinale $D$, et al. Prognostic value of troponin I in cardiac risk stratification of cancer patients undergoing high-dose chemotherapy. Circulation. 2004;109(22): 2749-54.

21. Lipshultz SE, et al. Predictive value of cardiac troponin T in pediatric patients at risk for myocardial injury. Circulation. 1997;96(8):2641-8.

22. Kistorp C, et al. N-terminal pro-brain natriuretic peptide, C-reactive protein, and urinary albumin levels as predictors of mortality and cardiovascular events in older adults. JAMA. 2005;293(13):1609-16.

23. Kragelund $\mathrm{C}$, et al. N-terminal pro-B-type natriuretic peptide and longterm mortality in stable coronary heart disease. N Engl J Med. 2005; 352(7):666-75.

24. Colan SD, et al. Developmental modulation of myocardial mechanics: ageand growth-related alterations in afterload and contractility. J Am Coll Cardiol. 1992;19(3):619-29. 
25. Lipshultz SE, et al. Changes in cardiac biomarkers during doxorubicin treatment of pediatric patients with high-risk acute lymphoblastic leukemia: associations with long-term echocardiographic outcomes. J Clin Oncol. 2012;30(10):1042-9.

26. Rusconi PG, et al. Serial measurements of serum NT-proBNP as markers of left ventricular systolic function and remodeling in children with heart failure. Am Heart J. 2010;160(4):776-83.

27. Gray RJ. A class of K-sample tests for comparing the cumulative incidence of a competing risk. Ann Stat. 1988;16(3):14.

28. Amigoni $\mathrm{M}$, et al. Low anthracyclines doses-induced cardiotoxicity in acute lymphoblastic leukemia long-term female survivors. Pediatr Blood Cancer. 2010;55(7):1343-7.

29. Silber $\mathbf{J H}$, et al. Increased risk of cardiac dysfunction after anthracyclines in girls. Med Pediatr Oncol. 1993;21(7):477-9.

30. Asselin BL, et al. Cardioprotection and safety of dexrazoxane in patients treated for newly diagnosed T-cell acute lymphoblastic leukemia or advanced-stage lymphoblastic non-Hodgkin lymphoma: a report of the Children's oncology group randomized trial pediatric oncology group 9404. J Clin Oncol. 2016;34(8):854-62.

31. Lipshultz SE, et al. The effect of dexrazoxane on myocardial injury in doxorubicin-treated children with acute lymphoblastic leukemia. N Engl J Med. 2004;351(2):145-53.

32. Lipshultz SE, et al. Assessment of dexrazoxane as a cardioprotectant in doxorubicin-treated children with high-risk acute lymphoblastic leukaemia: long-term follow-up of a prospective, randomised, multicentre trial. Lancet Oncol. 2010;11(10):950-61.

33. Wexler $\mathrm{LH}$, et al. Randomized trial of the cardioprotective agent ICRF-187 in pediatric sarcoma patients treated with doxorubicin. J Clin Oncol. 1996;14(2): 362-72.

34. Seidman A, et al. Cardiac dysfunction in the trastuzumab clinical trials experience. J Clin Oncol. 2002;20(5):1215-21.

35. Moja $L$, et al. Trastuzumab containing regimens for early breast cancer. Cochrane Database Syst Rev. 2012;(4):CD006243.

36. Kim IH, et al. Cardioprotective effect of dexrazoxane in patients with HER2positive breast cancer who receive anthracycline based adjuvant chemotherapy followed by trastuzumab. J Breast Cancer. 2017;20(1):82-90.

37. Keefe DL. Trastuzumab-associated cardiotoxicity. Cancer. 2002;95(7):1592-600.

38. Fiuza M. Cardiotoxicity associated with trastuzumab treatment of HER2+ breast cancer. Adv Ther. 2009;26(Suppl 1):S9-17.

39. Teepen JC, et al. Long-term risk of subsequent malignant neoplasms after treatment of childhood cancer in the DCOG LATER study cohort: role of chemotherapy. J Clin Oncol. 2017;35(20):2288-98.

40. Chow EJ, et al. Late mortality after dexrazoxane treatment: a report from the Children's oncology group. J Clin Oncol. 2015;33(24):2639-45.

41. Barry EV, et al. Absence of secondary malignant neoplasms in children with high-risk acute lymphoblastic leukemia treated with dexrazoxane. J Clin Oncol. 2008;26(7):1106-11.

42. Seif $A E$, et al. Dexrazoxane exposure and risk of secondary acute myeloid leukemia in pediatric oncology patients. Pediatr Blood Cancer. 2015;62(4): 704-9.

43. Vrooman LM, et al. The low incidence of secondary acute myelogenous leukaemia in children and adolescents treated with dexrazoxane for acute lymphoblastic leukaemia: a report from the Dana-Farber Cancer Institute ALL consortium. Eur J Cancer. 2011;47(9):1373-9.

44. EMA. Outcome of a procedure under Article 13 of Regulation (EC) No. 1234/ 2008. 2017. Available from: http://www.ema.europa.eu/ema/index.jsp?curl= pages/medicines/human/referrals/Cardioxane/human_referral_000421. jsp\&mid=WC0b01ac05805c516f. [cited 2017 10/13/17]

45. Chow EJ, et al. Effect of dexrazoxane on heart function among long-term survivors of childhood leukemia and lymphoma: a report from the Children's oncology group (COG). Blood. 2016;128(22):696.

46. Bhakta N, et al. The cumulative burden of surviving childhood cancer: an initial report from the St Jude lifetime cohort study (SJLIFE). Lancet. 2017.

47. Bates $\mathrm{M}$, et al. A pharmacoeconomic evaluation of the use of dexrazoxane in preventing anthracycline-induced cardiotoxicity in patients with stage IIIB or IV metastatic breast cancer. Clin Ther. 1997;19(1):167-84.

48. Hutchins KK, et al. Prevention of cardiotoxicity among survivors of childhood cancer. Br J Clin Pharmacol. 2017;83(3):455-65.

\section{Publisher's Note}

Springer Nature remains neutral with regard to jurisdictional claims in published maps and institutional affiliations.

\section{Ready to submit your research? Choose BMC and benefit from:}

- fast, convenient online submission

- thorough peer review by experienced researchers in your field

- rapid publication on acceptance

- support for research data, including large and complex data types

- gold Open Access which fosters wider collaboration and increased citations

- maximum visibility for your research: over $100 \mathrm{M}$ website views per year

At $\mathrm{BMC}$, research is always in progress.

Learn more biomedcentral.com/submissions 